\title{
Special AT-rich sequence-binding protein-1 participates in the maintenance of breast cancer stem cells through regulation of the Notch signaling pathway and expression of Snail1 and Twist1
}

\author{
ZHENGKUI SUN $^{1}$, CHAO ZHANG ${ }^{2}$, XUESEN ZOU $^{1}$, GUIXIANG JIANG ${ }^{1}$, \\ ZONGQUAN XU ${ }^{1}$, WENTING $\mathrm{LI}^{2}$ and HUI XIE ${ }^{2}$ \\ ${ }^{1}$ Department of Breast Surgery, Jiangxi Cancer Hospital; ${ }^{2}$ Department of Clinical Medicine, \\ Medical School of Nanchang University, Nanchang, Jiangxi 330029, P.R. China
}

Received January 12, 2014; Accepted November 12, 2014

DOI: $10.3892 / \mathrm{mmr} .2015 .3192$

\begin{abstract}
The stem cell populations in cancerous tissues and cell lines vary widely and are often associated with aggressive cases of breast cancer. Despite research on the topic, the mechanism underlying the regulation of the breast cancer stem cell (BCSC) population within tumors remains to be fully elucidated. To investigate the function of special AT-rich sequence-binding protein-1 (SATB1) in the maintenance of the BCSC population, SATB1 was overexpressed with lentivirus in MCF-7 cells or knocked down with shRNA-lentivirus in BT-549 cells. The effects of SATB1 overexpression or knockdown on mammosphere formation, the size of the of BCSC population, cell invasion and tumorigenesis were investigated. Activation of the Notch signaling pathway and expression of Snaill and Twistl were also examined in the cells. Overexpression of SATB1 in MCF-7 cells was observed to increase mammosphere formation, the size of the BCSC population, cell invasion and tumorigenesis, accompanied by an increase in the activation of Notch signaling and expression levels of Snail1 and Twist1. Conversely, knockdown of SATB1 in BT-549 cells produced the opposite effects. The results indicated that expression of SATB1 may increase the size of the BCSC population via the activation of the Notch signaling pathway and by increasing expression levels of Snaill and Twist1.
\end{abstract}

\section{Introduction}

Breast cancer is a notable cause of morbidity and mortality in females, and is associated with a high incidence of recur-

Correspondence to: Professor Zhengkui Sun, Department of Breast Surgery, Jiangxi Cancer Hospital, 519 East Beijing Road, Nanchang, Jiangxi 330029, P.R. China

E-mail: sunzhengkui616@163.com

Key words: breast cancer, SATB1, cancer stem cells, mammosphere, epithelial-mesenchymal transition rence and treatment failure (1). Growing evidence suggests that in patients with breast cancer, tumor metastasis and poor clinical outcome may be due to the evasion of the effects of systemic therapies by a small subset of stem-like cells, termed breast cancer stem cells (BCSCs) (2). In severe combined immunodeficiency (SCID) model mice, BCSCs are identified by the presence of a combination of $\mathrm{CD} 44^{+} / \mathrm{CD} 24^{-}$, aldehyde dehydrogenase 1 activity, mammosphere formation and tumorigenicity $(3,4)$. The BCSC population varies widely among cancerous tissues and cell lines and is often associated with aggressive types of breast cancer (5). Alterations in critical signaling pathways such as Notch, Wnt/ $\beta$-catenin and Hedgehog allow stem cells to undergo uncontrolled proliferation (6,7). Notably, previous studies have suggested that the activation of Notch promotes the expansion of BCSCs. In cells with enriched BCSC markers, Notch-1 signaling activity was observed to increase 4-fold while Notch-4 signaling activity increased 8-fold $(8,9)$. In addition, BCSCs are able to undergo epithelial-mesenchymal transition (EMT), a process that is involved in the facilitation of breast cancer progression (10-12). It has been suggested that Snail1 activity is required for EMT initiation, whereas Twist1 serves a role in the maintenance of EMT in mammary epithelial and breast cancer cells $(10,13)$.

Previous studies have suggested that special AT-rich sequence-binding protein-1 (SATB1), which functions as a genome organizer, is crucial in the progression of breast cancer towards metastasis. Deregulation of SATB1 in malignant cells alone, in lieu of multiple successive genomic aberrations, is sufficient to alter the expression of a large number of genes required for the progression of cancer to metastasis $(14,15)$. In the current study, the effects of SATB1 overexpression or knockdown were investigated on the stem cell populations in the breast cancer cell lines MCF-7 or BT-549, respectively, by assessment of in vitro mammosphere formation and CD $44^{+} / \mathrm{CD} 21^{-}$expression, and observation of tumor formation in SCID mice. A previous study demonstrated that the number of mammospheres generated was an indirect measure of mammary stem cell self-renewal; mammosphere size was representative of progenitor cell proliferation; and that the CD $44^{+} / \mathrm{CD} 24$ - population of breast cancer cells display characteristics of stem cells (3). Thus, these factors were investigated 
in the present study. In order to determine a possible mechanism of SATB1 in maintaining the BCSC population, the expression levels of Notch1, Notch4, Hes1, Snail1 and Twist1 were examined in the MCF-7 and BT-549 cell lines.

\section{Materials and methods}

Lentiviral construction and cell transfection. All lentiviral constructs were prepared by Shanghai GeneChem Co., Ltd. (Shanghai, China). Lentivirus GV287-SATB1 and Lentivirus GV115-SATB1-shRNA transfection was conducted in accordance with the manufacturer's instructions (GeneChem, Co., Ltd). The human SATB1 cDNA was subcloned into the GV287 lentiviral vector (http://www.genechem.com. cn/Zaiti.aspx?zt=GV287) and the human SATB1-small hairpin (sh)RNA targeted to SATB1 or negative control (NC)shRNA (GeneChem, Co., Ltd) were subcloned into the GV115 lentiviral vector (http://www.genechem.com.cn/Zaiti. aspx?zt=GV115). Subsequently, the lentivirus vector and packaging plasmid mixes were transfected into HEK293T cells (American Type Culture Collection, Manassas, VA, USA) using Lipofectamine ${ }^{\circledR} 2000$ (Invitrogen Life Technologies, Carlsbad, CA, USA). Following 48-h transfection, the Dulbecco's modified Eagle's medium (DMEM; Gibco, Life Technologies, Grand Island, NY, USA) was harvested and filtered. Subsequent to confirmation via restriction digestion with AgeI restriction enzyme (New England Biolabs, Beverly, MA, USA) and DNA sequencing performed by GeneChem using a pyrosequencing method, large-scale GV287-SATB1, GV115-SATB1-shRNA and GV115-NC-shRNA viruses were produced and used for the transfection into the breast cancer cell lines.

Cell lines culture and mammosphere assay. The human breast cancer cell lines MCF-7 and BT-549 were obtained from the American Type Culture Collection (Manassas, VA, USA) and maintained in high-glucose DMEM (GE Healthcare Life Sciences, Logan, UT, USA) supplemented with $10 \%$ fetal bovine serum (FBS; GE Healthcare Life Sciences) at $37^{\circ} \mathrm{C}$ in $5 \% \mathrm{CO}_{2}$. For transfection, the MCF-7 cells were infected with the GV287-SATB1 or the control lentivirus GV287, whereas BT-549 cells were infected with GV115-SATB1-shRNA or lentivirus GV115-NC-shRNA. Following 12 16 h incubation, the viruses were removed and replaced with fresh DMEM. For the mammosphere experiments, single-cell suspensions of the breast cancer cells were plated on ultra-low attachment plates (Corning Inc., Corning, NY, USA) at a density of $1 \times 10^{4}$ cells/well in DMEM supplemented with $2 \%$ (v/v) B-27 (Invitrogen Life Technologies) and $20 \mathrm{ng} / \mathrm{ml}$ EGF and bFGF (Peprotech, Inc., Rocky Hill, NJ, USA). Fresh medium was added to the culture every $48 \mathrm{~h}$ and images of the resultant non-adherent mammospheres were captured in triplicate using a digital camera (Coolpix 990; Nikon Corp., Tokyo, Japan) on day 10. The diameters of the spheres were measured using Photoshop CS5 (Adobe Systems, Inc., San Jose, CA, USA) and the average sphere sizes in each of the 10 fields were calculated. Quantification of the efficiency of sphere formation involved counting the mammospheres under a CK40 light microscope (Olympus Corp., Tokyo, Japan) at a magnification of x10 and recording the number of mammospheres/spheres formed in the 96 wells divided by the original number of single cells seeded, expressed as a percentage.

Flow cytometric analysis. Adherent cells were lifted using $0.25 \%(\mathrm{v} / \mathrm{v})$ trypsin and washed with phosphate-buffered saline (PBS) (Spectrum Chemical (Shanghai) Co., Ltd, Shanghai, China), while mammosphere cells were collected via centrifugation for $5 \mathrm{~min}$ at $300 \mathrm{x} \mathrm{g}$ with a XKA-2200 centrifuge (Xiangyi Group, Changsha, China), dissociated using trypsin and washed with PBS. The dissociated cells were resuspended to a final concentration of $5 \times 10^{6}$ cells $/ \mathrm{ml}$ in PBS and $5 \times 10^{5}$ cells $(100 \mu \mathrm{l})$ were incubated with $0.5 \sim 1.0 \mu \mathrm{g}$ phycoerythrin (PE)-conjugated mouse anti-human/mouse CD44 (12-0441) and allophycocyanin (APC)-conjugated mouse anti-human CD24 antibodies (17-0247) or isotype matched control antibodies (1:200; BD Biosciences, Heidelberg, Germany) at $4^{\circ} \mathrm{C}$ for $60 \mathrm{~min}$. The cells were then washed twice with ice-cold PBS and collected by centrifugation for $5 \mathrm{~min}$ at $300 \mathrm{x} \mathrm{g}$ for flow cytometric analysis using the FACSAria II (BD Biosciences) with FACSDiva software (BD Biosciences) indicating APC (CD24) fluorescence on the $\mathrm{x}$-axis and $\mathrm{PE}$ (CD44) fluorescence on the y-axis.

Invasion assays. A Transwell system ( 24 wells; $8 \mu \mathrm{m}$ pore size; BD Biosciences) coated with $2 \mathrm{mg} / \mathrm{ml}$ basement membrane Matrigel (BD Biosciences) was used for the in vitro invasion assays. A total of $1 \times 10^{5}$ cells were suspended in serum-free DMEM (Gibco) in the upper chamber of each well, while the lower chamber of each well was filled with $750 \mu 1$ DMEM supplemented with $10 \%$ FBS. Subsequent to suspension for $24 \mathrm{~h}$, the filters were fixed with methanol and stained with $0.1 \%$ crystal violet (Spectrum Chemical (Shanghai) Co., Ltd). The number of cells in at least five randomly selected microscope fields (the filter was divided into 16 microscopic fields and five were selected using a random number method) were then counted and underwent statistical analysis.

Implantation of cells in SCID model mice. MCF-7 cells infected with GV287 or GV287-SATB1 lentivirus, or BT-549 cells infected with GV115-SATB1-shRNA or GV115-NC-shRNA lentivirus, were washed in PBS and then injected into the mammary fat pad of 5-week-old female SCID mice anesthetized with pentobarbital sodium (Sigma-Aldrich) administered via intraperitoneal injection $(40 \mathrm{mg} / \mathrm{kg})$. To test the success rates of engraftment, 10 mice of each group were respectively injected with $10^{3}, 10^{4}$ and $10^{5}$ cells, and to test engraftment size, 6 mice of each group were injected with $10^{6}$ cells. The mice (20-30 g), obtained from SLRC Laboratory Animals (Shanghai, China) were maintained in laminar flow rooms under constant temperature and humidity and received estradiol supplementation $(0.4 \mathrm{mg} / \mathrm{kg}$; Novo Nordisk, Copenhagen, Denmark) every week subsequent to cell injection. Mice were inspected for tumor appearance daily by observation and palpation for 12 weeks subsequent to cell injection. Tumor volumes were calculated using the formula $\mathrm{V}=\mathrm{L}\left(\mathrm{W}^{2}\right) / 2$, in which $\mathrm{L}$ indicates length and $\mathrm{W}$ indicates tumor width. At the conclusion of the experiments, mice were sacrificed by cervical dislocation and the presence of each tumor nodule was confirmed by necropsy. Experimental protocols were approved by the Ethics Committee for Animal Experimentation of Jiangxi Cancer Hospital (Nanchang, China). 
A

MCF-7

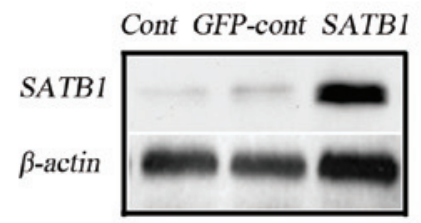

B $\quad B T-549$

SATB

$\beta$-actin

Cont sh-NC sh-SATBI

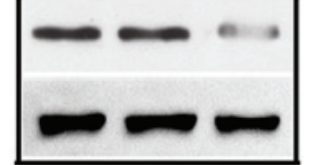

C $\quad M C F-7$

Cont

GFP-cont

SATB1
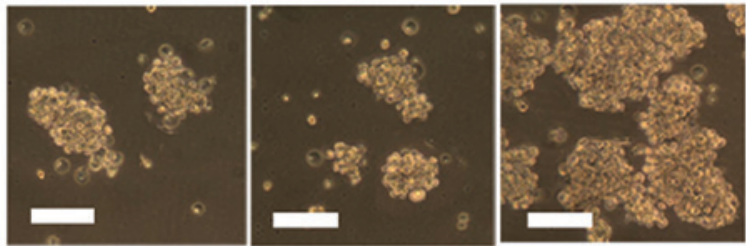

D

$\mathbf{E}$
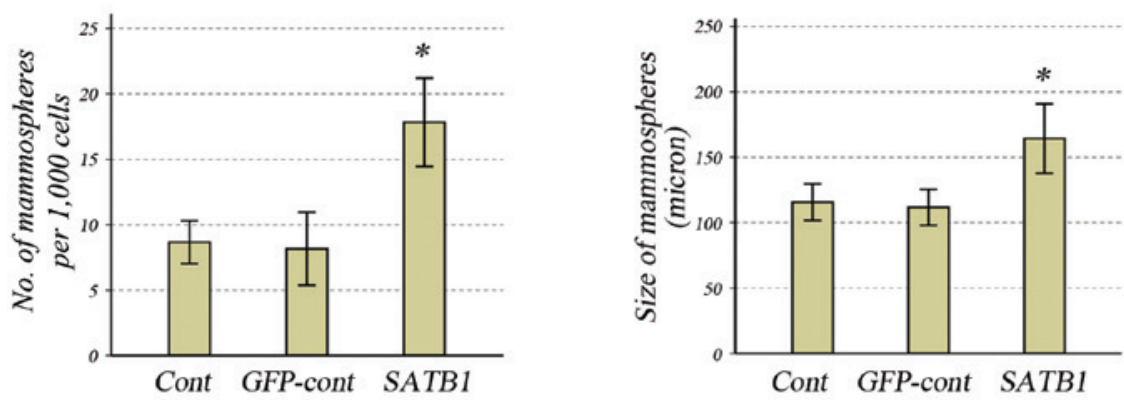

F $\quad$ BT-549

Cont

sh-NC

sh-SATB1

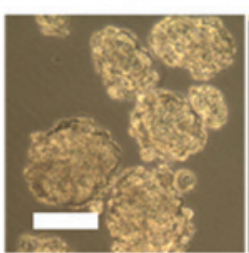

G

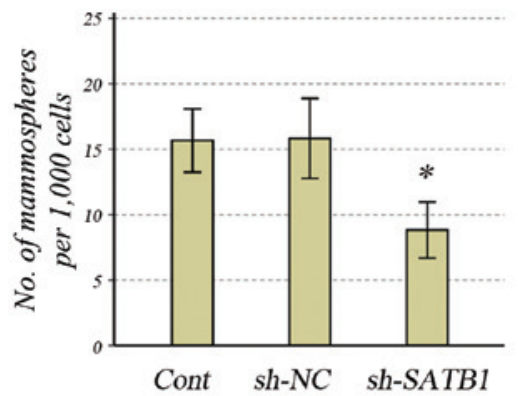

H

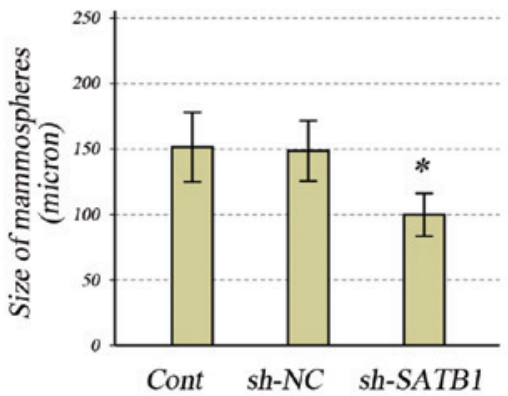

Figure 1. Effects of SATB1 expression on mammosphere formation in human breast cancer cell lines. Western blot analysis for SATB1 expression in (A) MCF-7 and (B) BT-549 cells. (C) Light microscope images of the MCF-7 cells (magnification, x200; scale bar, $10 \mu \mathrm{m}$ ). (D) Number of and (E) size of mammospheres in SATB1-expressing MCF-7 cells compared with the controls. (F) Light microscope images of the BT-549 cells (magnification, x200; scale bar, $10 \mu \mathrm{m}$ ). (G) Number of and $(\mathrm{H})$ size of mammospheres in SATB1-knockdown BT-549 cells compared with the controls. All data are presented as the mean \pm standard deviation of two independent experiments in triplicate, ${ }^{*} \mathrm{P}<0.01$ vs. controls. SATB1, special AT-rich sequence-binding protein-1; cont, control; GFP, green fluorescent protein; sh, small hairpin; NC, negative control.

Immunoblotting. Cells and tumor tissues were lysed in a laemmli buffer (Bio Rad Laboratories, Inc., Hercules, CA, USA), boiled and loaded onto SDS (Thermo Fisher
Scientific, Rockford, IL, USA)-polyacrylamide gels (Energy Chemical, Shanghai, China). Following electrophoresis (Bio Rad Laboratories, Inc.), proteins were transferred onto 

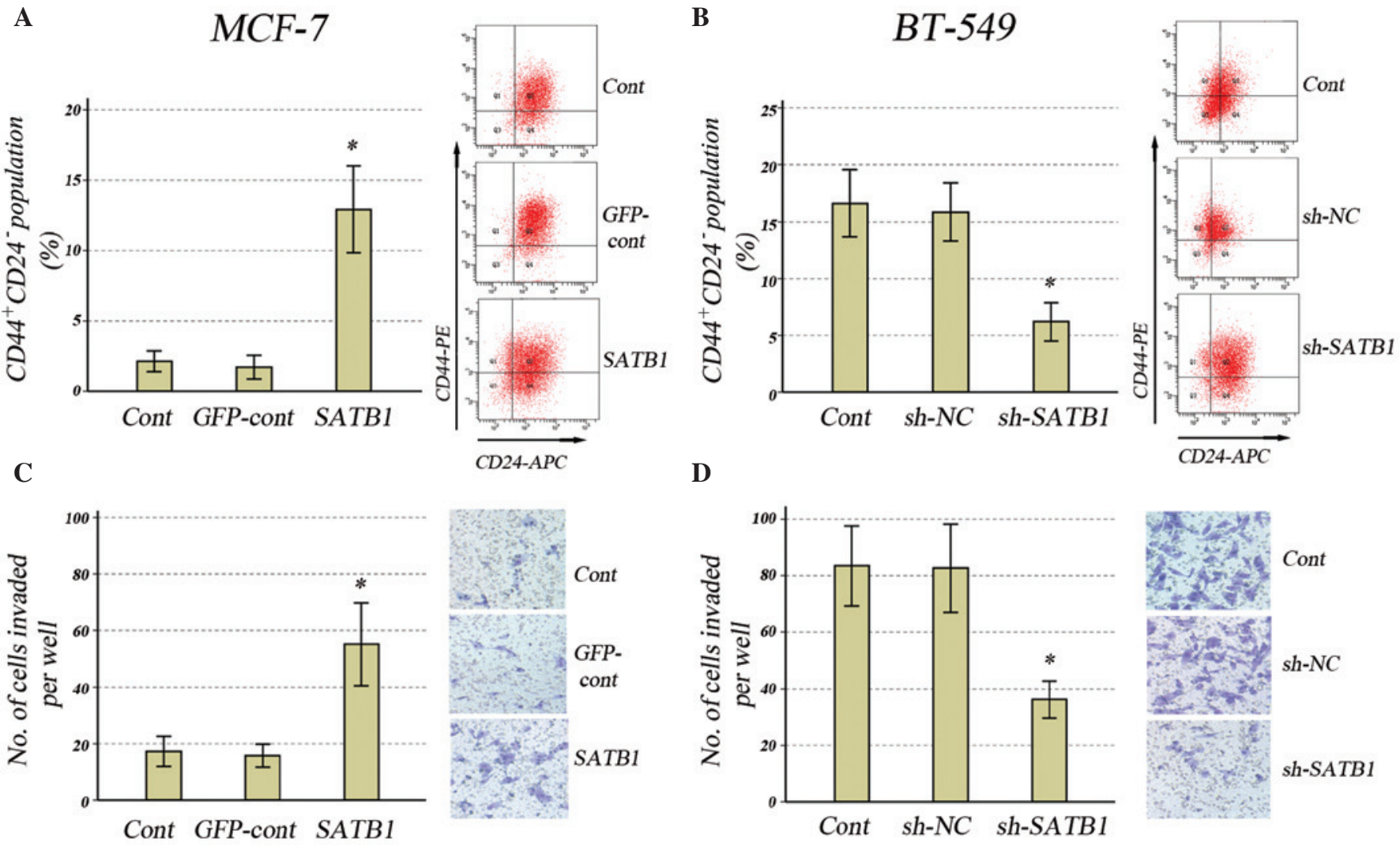

Figure 2. Effects of SATB1 expression on the CD44 ${ }^{+} / \mathrm{CD} 24^{-}$population and invasive properties of human breast cancer cell lines. Populations of CD44 ${ }^{+} / \mathrm{CD} 24$ in (A) MCF-7 cells with SATB1 overexpression and (B) BT-549 cells with SATB1 knockdown, with representative images from the CD44/CD24 flow cytometric analysis. Invasiveness in (C) MCF-7 cells with SATB1 overexpression and (D) BT-549 cells with SATB1 knockdown, and representative images taken from the invasion assays (magnification, $\mathrm{x} 20$ ). All data are presented as the mean \pm standard deviation of two independent experiments in triplicate; ${ }^{*} \mathrm{P}<0.001$ vs. controls. SATB1, special AT-rich sequence-binding protein-1; cont, control; GFP, green fluorescent protein; sh, small hairpin; NC, negative control.

nitrocellulose membranes (GenScript USA Inc., Piscataway, NJ, USA) using Trans-Blot SD Semi-Dry Electrophoretic Transfer Cell (Bio-Rad Laboratories, Inc.). Blots were incubated in Tris-buffered saline (TBS) (Spectrum Chemical (Shanghai) Co., Ltd) blocking buffer containing $2 \%$ milk for $1 \sim 2 \mathrm{~h}$ at room temperature and then with the mouse monoclonal anti-human antibodies against Notch1 (sc-373891), Hes1 (sc-166410), Snail1 (sc-271977) and Twist1 (sc-81417), as well as rabbit anti-human polyclonal antibodies against SATB1 (sc-28676) and Notch4 (sc-5594) (Santa Cruz Biotechnology, Inc., Santa Cruz, CA, USA) diluted 1:200 in TBS with Tween (TBST; containing $0.1 \%$ Tween-20 and 2\% bovine serum albumin; Spectrum Chemical (Shanghai) Co., Ltd) overnight at $4^{\circ} \mathrm{C}$. Subsequently, blots were washed and incubated with the appropriate secondary antibodies (goat polyclonal anti-mouse IgG, sc-2005, and goat polyclonal anti-rabbit IgG, sc-2004; Santa Cruz Biotechnology Inc.) in TBST at a 1:100-1:200 dilution ratio and detected using the BeyoECL Plus Western Blotting Detection System (Beyotime, Haimen, China), in accordance with the manufacturer's instructions.

Histology and immunohistochemistry. Tumor tissues were fixed in $10 \%$ neutral buffered formalin ( $\mathrm{pH} 7.4$; Spectrum Chemical (Shanghai) Co., Ltd), embedded in paraffin (ApexBio Technology LLC, Houston, TX, USA), cut into 5- $\mu \mathrm{m}$ sections using a CUT6062 automatic paraffin slicing machine (SLEE Medical GmbH, Mainz, Germany) and stained with hematoxylin and eosin (Boster Biological Engineering Co., Ltd, Wuhan, China). For immunostaining, the sections were deparaffinized, rehydrated and stained with the anti-Ki67 antibody (Santa Cruz Biotechnology, Inc.) and VECTASTAIN Elite ABC Kit (Vector Laboratories, Burlingame, CA, USA) according to the manufacturer's instructions. For each slide examined, 1,000 cells were counted from 6 fields (randomly selected from 16 microscopic fields) at x20 magnification, and the percentage of Ki67-positive cells was calculated from the number of total cells. The number of Ki67-positive tumor cells in 100 tumor cells determined the Ki67 proliferation index.

Statistical analysis. Statistical analysis was performed using SPSS, version 19.0 (IBM SPSS, Armonk, NY, USA). Fisher's exact test was used to determine associations between SATB1 expression and the success rates of engraftment. The differences in the means of the groups were analyzed with one-way analysis of variance or independent-samples t-test. $\mathrm{P}<0.05$ was considered to indicate a statistically significant difference.

\section{Results}

Knockdown or overexpression of SATB1 reduces or increases the capacity for mammosphere formation in breast cancer cells, respectively. To examine the role of SATB1 in maintaining the BCSC population, the lentivirus GV287-SATB1 was selected to overexpress SATB1 in MCF-7 breast cancer 
A

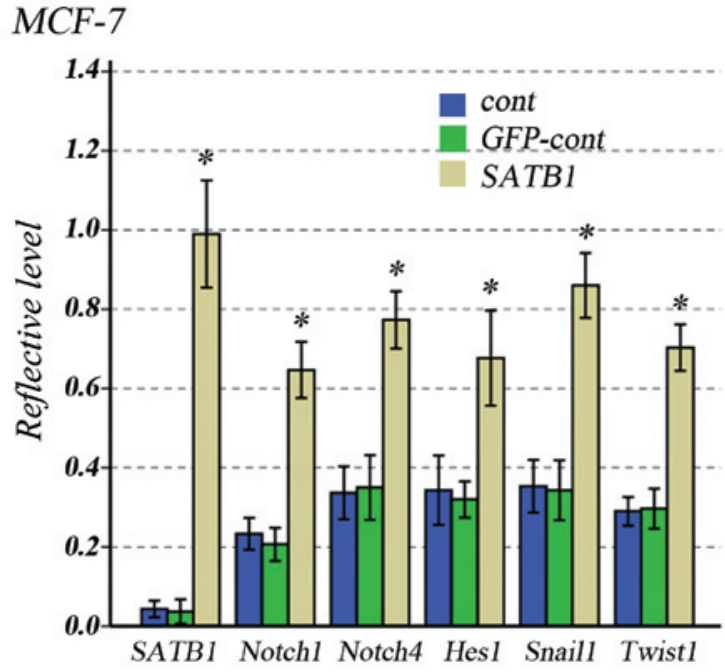

C

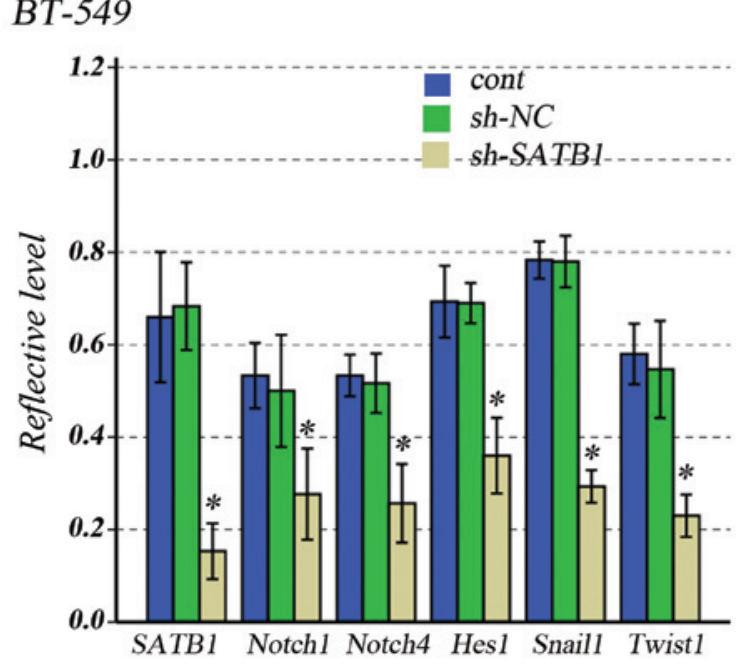

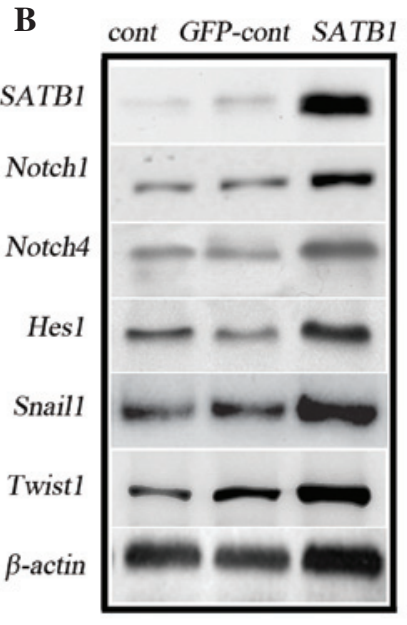

D cont sh-NC sh-SATB1

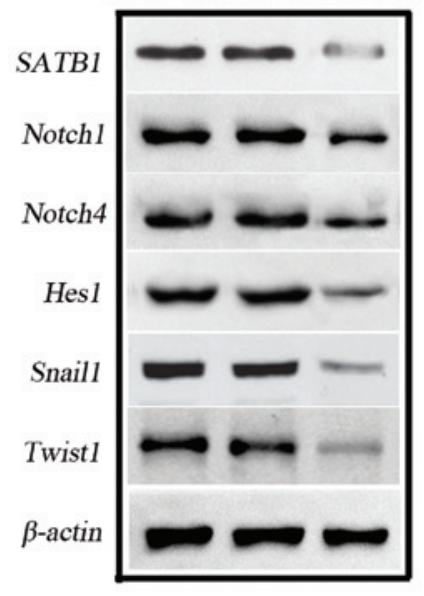

Figure 3. Effects of increased or reduced SATB1 expression on Notch1, Notch4, Hes1, Snail1 and Twist1 expression levels in human breast cancer cells. (A) Graph and (B) representative western blot of SATB1, Notch1, Notch4, Hes1, Snail1 and Twist1 expression levels in SATB1-overexpressing and control MCF-7 cells. (C) Graph and (D) representative western blot of SATB1, Notch1, Notch4, Hes1, Snail1 and Twist1 expression levels in SATB1-knockdown and control BT-549 cells. All data are presented as the mean \pm standard deviation of experiments in triplicate; ${ }^{*}$ P $<0.001$ vs. controls. SATB1, special AT-rich sequence-binding protein-1; cont, control; GFP, green fluorescent protein; sh, small hairpin; NC, negative control.

cells, while the shRNA-lentivirus GV115-SATB1-shRNA was selected to knockdown SATB1 in BT-549 cells. SATB1 overexpression or knockdown was confirmed by western blotting (Fig. 1A and B). When the MCF-7 cells transfected with GV287-SATB1 were cultured, the size and number of mammospheres significantly increased compared with the controls (Fig. 1C-E). In BT-549 cells transfected with GV115-SATB1-shRNA, the size and number of mammospheres was significantly reduced compared with the controls (Fig. 1F-H).

Effect of SATB1 expression on the $C D 44^{+} / C D 24^{-}$population and tumor invasiveness in breast cancer cells. In order to further determine the effects of SATB1 expression on the cancer stem cell population, the effects of SATB1 overexpression in MCF-7 cells and SATB1 knockdown in BT-549 cells on CD44 ${ }^{+} / \mathrm{CD} 24$ populations were investigated. In MCF-7 cells transfected with GV287-SATB1, SATB1 overexpression resulted in a significant increase in the $\mathrm{CD} 44^{+} / \mathrm{CD} 24^{-}$population (Fig. $2 \mathrm{~A}$ ). In BT-549 cells transfected with GV115-SATB1-shRNA, downregulation of SATB1 expression resulted in a significant reduction in the $\mathrm{CD} 44^{+} / \mathrm{CD} 24^{-}$population (Fig. $2 \mathrm{~B}$ ).

It has previously been suggested that tumor invasion and metastasis may be mediated by the cancer stem cell population (16,17), thus, the Matrigel-invading ability of MCF-7 cells infected with GV287-SATB1 or BT-549 cells infected with GV115-SATB1-shRNA was investigated. Overexpression of SATB1 resulted in a significant increase in invasiveness compared with control groups in the MCF-7 cells (Fig. 2C), whilst downregulation of SATB1 resulted in a significant reduction in invasiveness compared with control groups in BT-549 cells (Fig. 2D).

Knockdown or overexpression of SATB1 reduces or increases Notch1, Notch4, Hes1, Snaill and Twistl expression levels, respectively. To determine whether SATB1 affects Notch signaling pathways, the expression levels of genes involved in stem cell behavior, including Notch1, Notch4, Hes1, Snail1 and Twist1, were investigated in MCF-7 and BT-549 cells transfected with GV287-SATB1 and GV115-SATB1-shRNA, 
A

\section{$\mathrm{MCF}-7$}

\begin{tabular}{|c|c|c|c|}
\hline \multirow{2}{*}{$\begin{array}{c}\text { No. of } \\
\text { cells }\end{array}$} & \multicolumn{3}{|c|}{ Engraftments } \\
\cline { 2 - 4 } & GFP-cont & SATBI & $P$ \\
\hline $10^{5}$ & $6 / 10$ & $10 / 10$ & 0.04 \\
\hline $10^{4}$ & $1 / 10$ & $7 / 10$ & 0.01 \\
\hline $10^{3}$ & $0 / 10$ & $2 / 10$ & 0.24 \\
\hline
\end{tabular}

C

\section{BT-549}

\begin{tabular}{|c|c|c|c|}
\hline \multirow{2}{*}{$\begin{array}{c}\text { No. of } \\
\text { cells }\end{array}$} & \multicolumn{3}{|c|}{ Engraftments } \\
\cline { 2 - 4 } & sh-NC & sh-SATB1 & $P$ \\
\hline $10^{5}$ & $9 / 10$ & $5 / 10$ & 0.07 \\
\hline $10^{4}$ & $7 / 10$ & $1 / 10$ & 0.01 \\
\hline $10^{3}$ & $4 / 10$ & $0 / 10$ & 0.04 \\
\hline
\end{tabular}

B

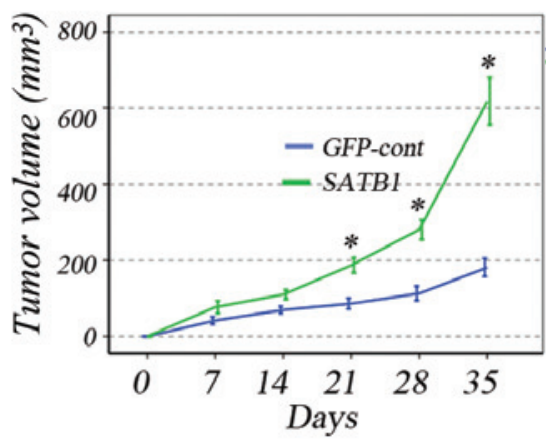

D

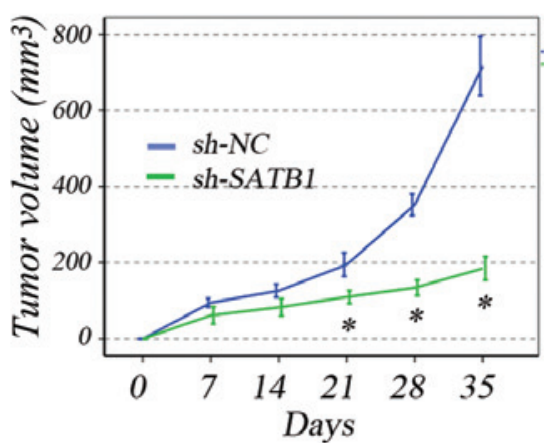

$\mathbf{E}$

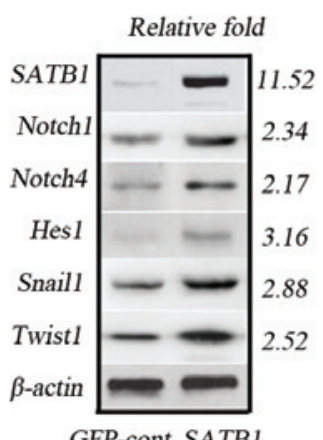

GFP-cont SATB1
F

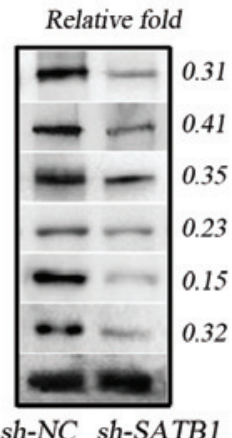

G

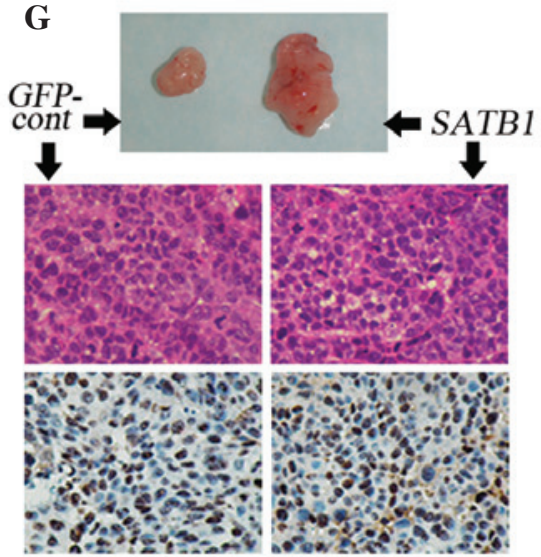

$\mathbf{H}$

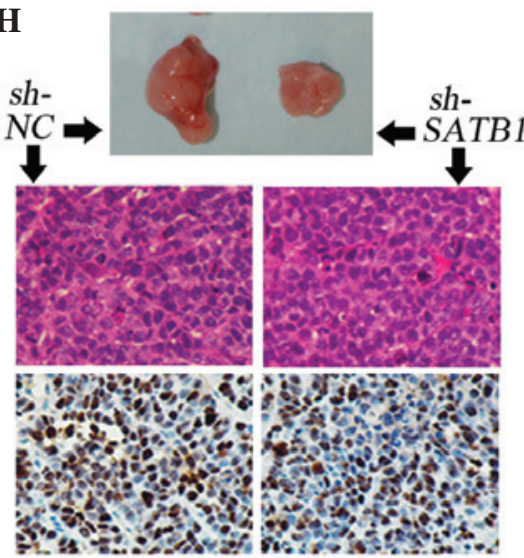

Figure 4. Effects of SATB1 expression on tumorigenicity and expression levels of Notch1, Notch4, Hes1, Snail1 and Twist1 in vivo. (A) The number of successful tumor engraftments correlated with the number of MCF-7-GFP-cont and MCF-7-SATB1 cells injected during serial dilution. (B) Tumor growth size subsequent to injection of 1x10 $\mathrm{MCF}-7-\mathrm{GFP}-\mathrm{cont}$ and MCF-7-SATB1 cells. (C) The number of successful tumor engraftments correlated with the number of BT-549-sh-NC and BT-549-sh-SATB1 cells injected during serial dilution. (D) Tumor growth size subsequent to injection of $1 \times 10^{6}$ BT-549-sh-NC and BT-549-sh-SATB1 cells. Representative immunoblot analysis and expression fold-change of SATB1, Notch1, Notch4, Hes1, Snail1 and Twist1 in (E) MCF-7-GFP-cont and MCF-7-SATB1-derived and (F) BT-549-sh-NC and BT-549-sh-SATB1-derived tumors relative to that of the control group. Representative images of xenografts, hematoxylin and eosin staining and immunohistochemical staining for Ki-67 xenografts, derived from injection of (G) $10^{6} \mathrm{MCF}-7-\mathrm{GFP}-\mathrm{cont}$ and MCF-7-SATB1 cells (magnification, x10) and (H) 10 BT-549-sh-cont and BT-549-sh-SATB1 cells (magnification, x10). Quantitative data are presented as the mean \pm standard deviation for six tumors in each group; ${ }^{~} \mathrm{P}<0.001$ vs. controls. SATB1, special AT-rich sequence-binding protein-1; GFP, green fluorescent protein; cont, control; sh, small hairpin; $\mathrm{NC}$, negative control; relative fold, expression fold-change relative to the control group.

respectively. Overexpression of SATB1 significantly increased the expression levels of Notch1, Notch4, Hes1, Snail1 and Twistl compared with control groups in MCF-7 cells (Fig. 3A), which can be observed by the results of the western blot analysis (Fig. 3B). Conversely, the downregulation of SATB1 reduced the expression of these genes compared with control groups in BT-549 cells (Fig. 3C), and can be observed by the results of the western blot analysis (Fig. 3D).

This suggests that SATB1 overexpression increases the size of the stem cell pool, and also activates Notch signaling pathways, increasing the expression levels of Snaill and Twist1, which drive EMT in breast cancer cells.
Effects of SATB1 expression on stem cell population, tumorigenicity and Notch1, Notch4, Hes1, Snaill and Twist1 expression levels in breast cancer cells in vivo. A subcutaneous serial dilution transplantation experiment was performed in female SCID mice using MCF-7 and BT-549 cells transfected with GV287-SATB1 and GV115-SATB1-shRNA, respectively. A notable observation was that following the subcutaneous implantation of $1 \times 10^{4}$ and $1 \times 10^{5}$ MCF-7 cells, SATB1 overexpression in the cells significantly increased the success of engraftments (Fig. 4A), and subsequent to the implantation of $1 \times 10^{6} \mathrm{MCF}-7$ cells, SATB1 overexpression significantly increased tumor size after 21 days (Fig. 4B). Following the 
subcutaneous implantation of $1 \times 10^{3}$ and $1 \times 10^{4}$ BT-549 cells, SATB1 depletion in the cells significantly reduced the success of engraftments (Fig. 4C), while subsequent to the subcutaneous implantation of $1 \times 10^{6}$ BT-549 cells, SATB1 knockdown significantly reduced tumor size after 21 days (Fig. 4D).

In agreement with the in vitro results, western blot analysis of the tumors revealed that in vivo overexpression of SATB1 in MCF-7 cells increased the expression levels of Notch1, Notch4, Hes1, Snail1 and Twist1 (Fig. 4E), whereas knockdown of SATB1 in BT-549 cells reduced the expression levels (Fig. 4F). Immunohistochemical analyses of the tumors revealed that the $\mathrm{Ki} 67$ proliferation index was comparable between MCF-7-GFP and MCF-7-SATB1 tumors (Fig. 4G), despite an increase in tumor size. In addition, the Ki67 proliferation index was comparable between BT-549-GFP and BT-549-sh-SATB1 tumors, despite a reduction in tumor size (Fig. 4H).

\section{Discussion}

Although SATB1 overexpression has been previously demonstrated to increase breast cancer invasion and metastasis, its effect on the stem cell population remains to be fully elucidated. In the current study, SATB1 was identified to expand the cancer stem cell population, accompanied by the activation of the Notch signaling pathway, which promotes cancer stem cell self-renewal and the expression of the Snail1 and Twist1 genes that drive EMT.

Overexpression of SATB1 in the breast cancer cell line MCF-7 was demonstrated to increase the population, which was measured by mammosphere formation and $\mathrm{CD} 44^{+} / \mathrm{CD} 24$ expression in vitro and formation of tumors in SCID model mice (in vivo). Downregulation of SATB1 in the breast cancer cell line BT-549 resulted in the opposite result. Previous studies have demonstrated that the subpopulation of breast cancer cells exhibit increased invasive properties (17). SATB1 overexpression increased the invasiveness of MCF-7 cells, and SATB1 knockdown reduced the invasiveness of BT-549 cells. These results suggest that SATB1 expression in breast cancer may increase the BCSC population, resulting in tumor progression.

Aberrant Notch signaling has been identified to be associated with the development and progression of breast cancer $(18,19)$; the Notch signaling pathway maintains the stemness of BCSCs (20). Previous studies have demonstrated that Notch signaling activity increased in BCSCs, as demonstrated by increased expression of the Notch receptors Notch1 and Notch4, in addition to Hes-1, an immediate downstream target of Notch signaling (8). In the current study, SATB1 overexpression in MCF-7 cells was demonstrated to significantly increase Notch1, Notch4 and Hes1 expression, whilst its knockdown in BT-549 cells resulted in the opposite effect. These data suggest that SATB1 mediates the BCSC population via the Notch signaling pathway.

Additionally, it has been hypothesized that the progression of the majority of carcinomas towards malignancy is associated with EMT, during which breast cancer cells transition to acquire stem cell-like properties $(21,22)$. In cancer EMT, signals emanate via factors, including transforming growth factor- $\beta$, hepatocyte growth factor, epidermal growth factor and hypoxia (23). The signals then converge upon a limited set of transcriptional repressors, including Snail1, Slug, ZEB1/2 and Twist1/2 (24,25). One study suggested that Snail1 activity is required for EMT initiation, whereas Twistl is involved in the maintenance of EMT during human breast cancer progression towards metastasis (13). In the current study, it was observed that Snaill and Twistl were upregulated in MCF-7 cells with SATB1 overexpression and inhibited in BT-549 cells with SATB1 knockdown. Therefore, in the present study, SATB1 expression was established to affect the number of BCSCs, in addition to their ability to propagate in conditions sustaining the undifferentiated cell state.

In conclusion, the current study suggests that the expression of SATB1 may increase the size of the BCSC population via the activation of Notch signaling, which is required for maintaining the stemness of BCSCs and increasing the expression level of Snaill and Twist1, which are required for EMT.

\section{Acknowledgements}

The present study was supported by the National Natural Science Foundation of China (grant nos. 81060179 and 81260329). The authors would like to thank Dr. Jianjun Xioang at the central laboratory of Jiujiang Medical College (Jiujiang, China) for his technical assistance.

\section{References}

1. Youlden DR, Cramb SM, Dunn NA, Muller JM, Pyke CM and Baade PD: The descriptive epidemiology of female breast cancer: an international comparison of screening, incidence, survival and mortality. Cancer Epidemiol 36: 237-248, 2012.

2. Zhou L, Jiang Y, Yan T, Di G, Shen Z, Shao Z and Lu J: The prognostic role of cancer stem cells in breast cancer: A meta-analysis of published literatures. Breast Cancer Res Treat 122: 795-801, 2010

3. Al-Hajj M, Wicha MS, Benito-Hernandez A, Morrison SJ and Clarke MF: Prospective identification of tumorigenic breast cancer cells. Proc Natl Acad Sci USA 100: 3983-3988, 2003.

4. Ginestier C, Hur MH, Charafe-Jauffret E, Monville F, Dutcher J, Brown M, Jacquemier J, Viens P, Kleer CG, Liu S, et al: ALDH1 is a marker of normal and malignant human mammary stem cells and a predictor of poor clinical outcome. Cell Stem Cell 1: 555-567, 2007.

5. Liu X, Johnson S, Liu S, Kanojia D, Yue W, Singh UP, Wang Q, Wang Q, Nie Q and Chen H: Nonlinear growth kinetics of breast cancer stem cells: implications for cancer stem cell targeted therapy. Sci Rep 3: 2473, 2013.

6. Kakarala M and Wicha MS: Cancer stem cells: Implications for cancer treatment and prevention. Cancer J 13: 271-275, 2007.

7. Takebe N, Harris PJ, Warren RQ and Ivy SP: Targeting cancer stem cells by inhibiting Wnt, Notch, and Hedgehog pathways. Nat Rev Clin Oncol 8: 97-106, 2011.

8. Grudzien P, Lo S, Albain KS, Robinson P, Rajan P, Strack PR, Golde TE, Miele L and Foreman KE: Inhibition of Notch signaling reduces the stem-like population of breast cancer cells and prevents mammosphere formation. Anticancer Res 30: 3853-3867, 2010.

9. Harrison H, Farnie G, Howell SJ, Rock RE, Stylianou S, Brennan KR, Bundred NJ and Clarke RB: Regulation of breast cancer stem cell activity by signaling through the Notch4 receptor. Cancer Res 70: 709-718, 2010.

10. Mani SA, Guo W, Liao MJ, et al: The epithelial-mesenchymal transition generates cells with properties of stem cells. Cell 133: 704-715, 2008.

11. Creighton CJ, Chang JC and Rosen JM: Epithelial-mesenchymal transition in tumor-initiating cells and its clinical implications in breast cancer. J Mammary Gland Biol Neoplasia 15: 253-260, 2010. 
12. Drasin DJ, Robin TP and Ford HL: Breast cancer epithelial-to-mesenchymal transition: examining the functional consequences of plasticity. Breast Cancer Res 13: 226, 2011.

13. Tran DD, Corsa CA, Biswas H, Aft RL and Longmore GD: Temporal and spatial cooperation of Snaill and Twist1 during epithelial-mesenchymal transition predicts for human breast cancer recurrence. Mol Cancer Res 9: 1644-1657, 2011.

14. Han HJ, Russo J, Kohwi Y and Kohwi-Shigematsu T: SATB1 reprogrammes gene expression to promote breast tumour growth and metastasis. Nature 452: 187-193, 2008.

15. Ordinario E, Han HJ, Furuta S, et al: ATM suppresses SATB1-induced malignant progression in breast epithelial cells. PLoS One 7: e51786, 2012.

16. Wicha MS: Cancer stem cells and metastasis: lethal seeds. Clin Cancer Res 12: 5606-5607, 2006.

17. Sheridan C, Kishimoto H, Fuchs RK, Mehrotra S, Bhat-Nakshatri P, Turner CH, Goulet R Jr, Badve S and Nakshatri H: CD44+/CD24breast cancer cells exhibit enhanced invasive properties: an early step necessary for metastasis. Breast Cancer Res 8: R59, 2006.

18. Pece S, Serresi M, Santolini E, Capra M, Hulleman E, Galimberti V,Zurrida S, Maisonneuve P, Viale G and Di Fiore PP: Loss of negative regulation by Numb over Notch is relevant to human breast carcinogenesis. J Cell Biol 167: 215-221, 2004.
19. Stylianou S, Clarke RB and Brennan K: Aberrant activation of notch signaling in human breast cancer. Cancer Res 66: $1517-1525,2006$.

20. Han J, Hendzel MJ and Allalunis-Turner J: Notch signaling as a therapeutic target for breast cancer treatment? Breast Cancer Res 13: 210, 2011.

21. Battula VL, Shi Y, Evans KW, et al: Ganglioside GD2 identifies breast cancer stem cells and promotes tumorigenesis. J Clin Invest 122: 2066-2078, 2012.

22. Battula VL,Evans KW,Hollier BG, et al: Epithelial-mesenchymal transition-derived cells exhibit multilineage differentiation potential similar to mesenchymal stem cells. Stem Cells 28: 1435-1445, 2010

23. Thiery JP, Acloque H, Huang RY and Nieto MA: Epithelial-mesenchymal transitions in development and disease. Cell 139: 871-890, 2009.

24. Peinado H, Olmeda D and Cano A: Snail, Zeb and bHLH factors in tumour progression: an alliance against the epithelial phenotype? Nat Rev Cancer 7: 415-428, 2007.

25. Yang MH, Hsu DS, Wang HW, et al: Bmil is essential in Twist1-induced epithelial-mesenchymal transition. Nat Cell Biol 12: 982-992, 2010. 